\title{
Q-switching of an erbium-doped fibre laser modulated by a Bragg grating fixed to a piezoelectric
}

\author{
R Duchowicz $^{1,2}$, N A Russo ${ }^{1}$, E Sicre ${ }^{3}$ and M V Andrés ${ }^{4}$ \\ ${ }^{1}$ Centro de Investigaciones Opticas, CIOp (CONICET, CIC), La Plata, Argentina \\ 2 Departamento de Física, Facultad Ciencias Exactas, UNLP, La Plata, Argentina \\ ${ }^{3}$ Universidad Argentina de la Empresa (UADE), Buenos Aires, Argentina \\ ${ }^{4}$ Departamento de Física Aplicada, Universitat de Valéncia, Burjassot, Spain \\ E-mail: ricardod@ciop.unlp.edu.ar(R Duchowicz)
}

Received 18 November 2002, in final form 10 March 2003

Published 22 August 2003

Online at stacks.iop.org/JOptA/5/S216

\begin{abstract}
The performance of the Q-switched erbium-doped fibre laser with two fibre Bragg gratings as cavity mirrors was theoretically analysed, employing a set of rate equations for the ion populations and the photon flux inside the cavity. The simulation considers a system where the pulsed laser emission is produced by the temporal modulation of a Bragg grating fixed to a piezoelectric and operating in the $1550 \mathrm{~nm}$ spectral region. The temporal evolution of different frequency components of the laser emission produced is governed by the instantaneous overlap between the two gratings. Theoretical results are in agreement with previously reported experimental values.
\end{abstract}

Keywords: Fibre laser, erbium, Bragg grating, Q-switching

\section{Introduction}

Erbium-doped fibre lasers (EDFLs) have a variety of potential applications, as sources for wavelength division multiplexing (WDM) and soliton communications systems, and in sensor devices, optical time domain reflectometry, non-linear phenomena studies and applications, etc. Continuous-wave, Q-switching and mode-locking laser systems constructed by using doped fibres have been demonstrated. High continuouswave powers $(>100 \mathrm{~W})$ have been achieved with fibre lasers by using the cladding pump technique. In the pulsed operation regime (the Q-switched mode), the energy storage that can be achieved within conventional single-mode erbium-doped fibre limits the maximum energy. The use of fibre Bragg gratings to develop the laser cavity allows one to operate the laser with a narrow linewidth because of the frequency-selective reflectivity of such gratings. They act as high-reflectivity mirrors for the laser emission wavelength, but are transparent to the pump radiation, allowing the obtaining of very low-loss resonators. This has the additional advantage that an all-fibrein-line laser system can be developed [1-5].

Recently, we reported the performance of a Q-switched erbium-doped fibre laser operating with two fibre Bragg gratings as cavity mirrors where the temporal modulation is performed fixing one of the gratings to a piezoelectric (PZT: $\left.\mathrm{Pb}\left(\mathrm{Zr}_{x} \mathrm{Ti}_{1-x}\right) \mathrm{O}_{3}\right)$ element $[6,7]$. Applying voltage pulses to the PZT, the laser cavity was modulated. By using this simple scheme, a Q-switched laser output has been generated with high laser efficiency of energy conversion. Q-switched laser pulses of $0.5 \mathrm{~W}$ peak powers were obtained pumping at 76 $\mathrm{mW}$ at $18.5 \mathrm{kHz}$ and an energy conversion efficiency of $26 \%$ was obtained. Typical laser emission had temporal widths of 2-3 $\mu \mathrm{s}$ and an optical bandwidth of $\sim 0.1 \mathrm{~nm}$. The results depend on the pumping level of the active medium. In particular, exciting the medium at pump powers larger than six times $P_{p, h}$, where $P_{p, h}$ is the pump power of the laser threshold, produces not an increase in the laser output but the generation of additional laser peaks. A similar scheme has been reported before, but its performance was quite poor [8].

In view of the importance of fibre lasers and of the necessity of having perfect knowledge of the device operation, it is important to obtain a correct description of the laser evolution. So, we analyse here the behaviour of the Qswitched laser by using a general theoretical model based on a homogeneous, three-level approximation of amplification in erbium and compare the results with the behaviour of our Q- 


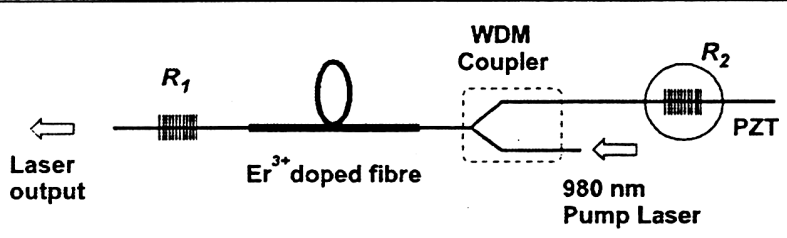

Figure 1. The laser scheme.

switched erbium fibre laser based on a modulation produced by a Bragg grating fixed to PZT. The laser cavity was simulated assuming that its wavelength (frequency) distribution has a different temporal modulation for each component.

\section{Theoretical model and results}

The system is shown schematically in figure 1. The laser resonator consists of a fibre WDM coupler, a segment of erbium-doped fibre and two fibre Bragg gratings acting as mirrors. In the previous experimental work, we employed as the gain medium $5.5 \mathrm{~m}$ of erbium-doped fibre containing $790 \mathrm{ppm} \mathrm{Er}^{3+}$ with a core diameter of $\sim 4.8 \mu \mathrm{m}$, a numerical aperture (NA) of 0.21 and a confinement factor $(\Gamma)$ of 0.59 . Excitation was produced at $0.98 \mu \mathrm{m}$. By means of the pumping action, population from the ${ }^{4} \mathrm{I}_{15 / 2}$ manifold (level 0 ) is transferred to the ${ }^{4} I_{11 / 2}$ level (level 2). Excited ions in the ${ }^{4} I_{11 / 2}$ state decays to the ${ }^{4} \mathrm{I}_{13 / 2}$ manifold (level 1 , with a lifetime of $\tau_{1} \approx 10 \mathrm{~ms}$ ) in tens of microseconds, mainly in a non-radiative way. Lasing action occurs in the ${ }^{4} \mathrm{I}_{13 / 2} \rightarrow{ }^{4} \mathrm{I}_{15 / 2}$ transition. In general, the populations are not uniform along the amplifying path. However, for the sake of simplicity we assume that there is uniform volume excitation of the doped fibre. It is also assumed that there is no excited state absorption at the pump wavelength.

In the theoretical comparison, the erbium-doped fibre laser is treated as a three-level homogeneous system. For the continuous laser regime and pumping into the $0.98 \mu \mathrm{m}$ absorption band, the population in the ${ }^{4} I_{11 / 2}$ manifold is negligible in relation to the population in the other levels $\left(\tau_{2} \ll \tau_{1}\right)$ and the theoretical model may be considered as a two-level approximation. This could not be so for a highrepetition-rate pulsed regime, where the pump period would be of the same order as $\tau_{2}$. The laser dynamics, in this case, can be described by the usual set of coupled rate equations for the photon number and the normalized populations corresponding to the active levels $[1,9-13]$ :

$$
\begin{aligned}
& \frac{\partial \Delta n}{\partial \tau}=\left(P-1-\sum_{m} a_{m}^{-} i_{m}\right)-\left(P+1+\sum_{m} a_{m}^{+} i_{m}\right) \Delta n \\
& \quad+n_{2}\left(1-P+\sum_{m} a_{m}^{-} i_{m}+\frac{\tau_{1}}{\tau_{2}}\right) \\
& \frac{\partial n_{2}}{\partial \tau}=P-\Delta n P-n_{2}\left(P+\frac{\tau_{1}}{\tau_{2}}\right) \\
& \frac{\partial i_{m}}{\partial \tau}=\left(b\left(a_{m}^{+} \Delta n+a_{m}^{-}\left(1-n_{2}\right)\right)-\gamma_{m}(\tau)\right) i_{m} \\
& n_{0}+n_{1}+n_{2}=1 \\
& \Delta n=n_{1}-n_{0}
\end{aligned}
$$

where $\tau=t / \tau_{1}$ with $\tau_{1} \approx 10 \mathrm{~ms}$ the decay time of the upper level of the active medium and $t$ the time; $i_{m}$ is the photon flux spectral density, $n_{0}, n_{1}, n_{2}$ are the ion populations corresponding to the ${ }^{4} \mathrm{I}_{15 / 2},{ }^{4} \mathrm{I}_{13 / 2}{ }^{4} \mathrm{I}_{11 / 2}$ levels normalized to the total $\mathrm{Er}^{3+}$ population and $\Delta n$ is the population inversion between the ${ }^{4} \mathrm{I}_{15 / 2}$ and ${ }^{4} \mathrm{I}_{13 / 2}$ levels, also normalized; $P$ represents the pumping rate normalized to $2 \tau_{\Gamma}^{-1} ; a_{m}^{+. ;}=$ $\tau_{1}\left(\sigma_{e, m} \pm \sigma_{a, m}\right)$ with $\sigma_{e}, \sigma_{a}$ the emission and absorption crosssections of the gain medium (constants within the Bragg grating influence range); $b=l N / T$ where $l$ is the gain medium length, $N$ the total ion concentration and $T$ the time taken for the light to make one cavity round trip. The decay rate of the laser light in the cavity is given by $\gamma_{m}(\tau)=-\gamma_{0} \ln \left(R_{1} R_{2}(\tau)\right)$, where $\gamma_{0}=\tau_{1} / T$ is a dimensionless constant, $R_{1}$ and $R_{2}$ are the mirror reflectivities (Bragg gratings) of the optical cavity and $m$ is the frequency index.

When the PZT is excited, the wavelength distribution of the reflecting grating fixed to it $\left(R_{2}\right)$ starts to oscillate in the spectrum with an amplitude depending of the applied voltage. In order to correlate the experimental behaviour of [7] with theoretical results obtained with this model, we have simulated the reflectivity of the practical gratings (which were characterized in that work by using an optical spectrum analyser) with a Gaussian distribution for the reflectivity of $R_{1}$ and a super-Gaussian for $R_{2}$ : Mathematically we express this as

$$
\begin{aligned}
R_{1}(\lambda) & =R_{01} \exp \left(-\left(\left(\lambda-\lambda_{1}\right) / \Delta \lambda_{1}\right)^{2}\right) \\
R_{2}(\lambda, \tau) & =R_{02} \exp \left(-\left(\left(\lambda-\lambda_{2}(\tau)\right) / \Delta \lambda_{2}\right)^{8}\right)
\end{aligned}
$$

with

$$
\lambda_{2}(\tau)=\lambda_{02}+\delta \lambda_{2} \sin \left(2 \pi f \tau_{1} \tau\right)
$$

where $\lambda$ is the wavelength, $\lambda_{1}, \lambda_{02}$ and $\Delta \lambda_{1}, \Delta \lambda_{2}$ are the central wavelength and the bandwidth of each grating reflectivity distribution, $\delta \lambda_{2}$ is the wavelength modulation amplitude and $f$ is the excitation frequency of the PZT. The set of coupled rate equations (1)-(5) under the cavity conditions given by equations (6)-(8) were solved numerically by using a personal computer with the help of a fourth-order Runge-Kutta integration method.

Typical parameters corresponding to a practical laser system of erbium-doped fibres were employed: $\tau_{2}=10^{-2} \mathrm{~s}$, $a_{\tilde{m}}=0.2 \times 10^{-27} \mathrm{~m}^{2} \mathrm{~s}, a_{m}^{+}=2.8 \times 10^{-27} \mathrm{~m}^{2} \mathrm{~s}, b=$ $1.4 \times 10^{32} \mathrm{~m}^{2} \mathrm{~s}^{-1}, \gamma_{0}=2 \times 10^{7}, R_{01} \equiv 0.75, \lambda_{1} \equiv$ $1540.3 \mathrm{~nm}, \Delta \lambda_{1}=0.12 \mathrm{~nm}, R_{02}=0.97, \lambda_{02}=1540.0 \mathrm{~nm}$, $\Delta \lambda_{2} \equiv 0.38 \mathrm{~nm}, \delta \lambda_{2}=0.4 \mathrm{~nm}$ and $f=18.5 \mathrm{kHz}$. A value of 590 was taken for the ratio between the time decays $\left(\tau_{1} / \tau_{2}\right)$. When a modulated voltage is applied to the PZT, the temporal distribution of $R_{2}$ shifts in wavelength as given by equation (6), overlapping periodically with the reflectivity distribution of $R_{1}$ (figure 2). The amplitude of this displacement is, in principle, dependent on the PZT voltage input. The overlap time depends on this amplitude, the PZT frequency modulation and the spectral widths of the two gratings.

The evolution of the cavity factor $\left(R_{1} R_{2}\right)$ determines the laser operation. Figure 3 shows the wavelength dependence on the temporal evolution of this product. As can be observed, the cavity behaves differently for each laser component, shifting in time (in this case), for the positive edge of the distribution, from lower- to larger-wavelength components. The direction depends on the initial spectral positions of the two gratings. The influence of these temporal differences depends on the modulation amplitude and frequency. 


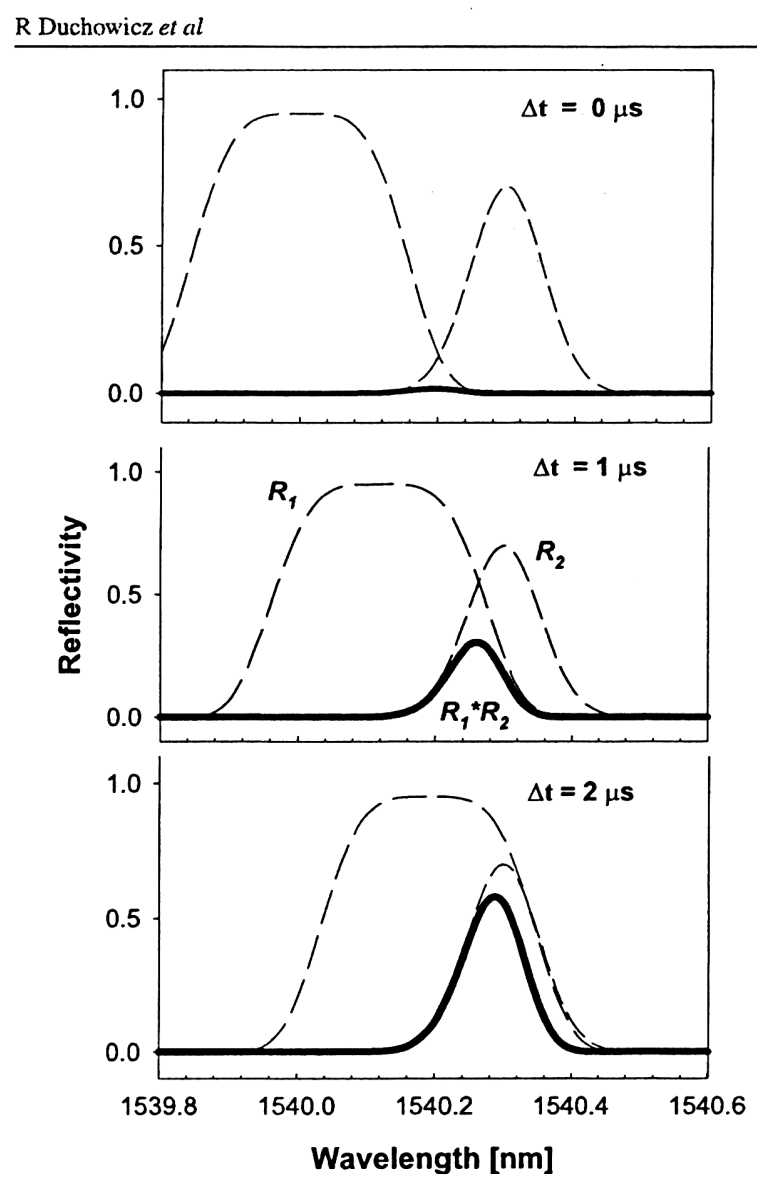

Figure 2. The spectral dependence of the temporal modulation.

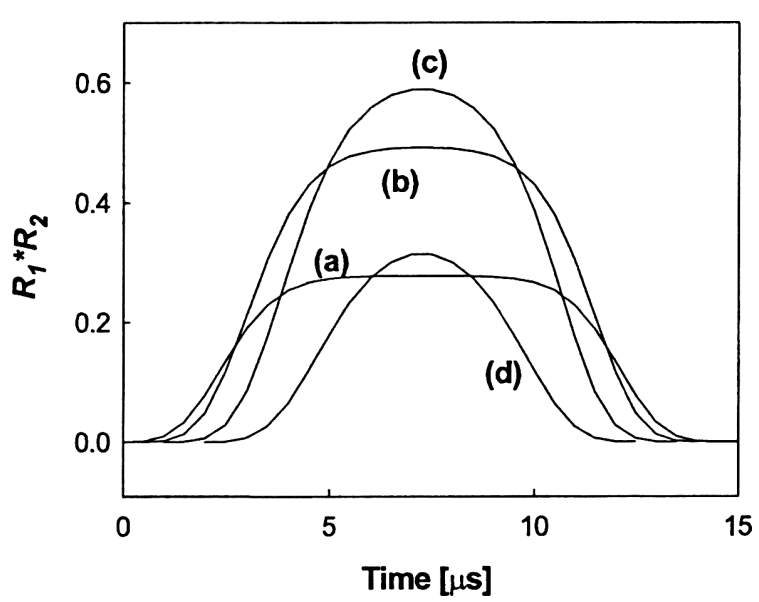

Figure 3. Spectral sweeping of the laser cavity. The spectral distributions correspond to: (a) 1540.23 , (b) 1540.26 , (c) 1540.30 and (d) $1540.34 \mathrm{~nm}$, respectively.

Figure 4 shows: (a) the PZT modulation voltage with a frequency $f_{0} \equiv 18.5 \mathrm{kHz}$, (b) the population inversion for the laser transition, (c) the population of the ${ }^{4} I_{11 / 2}$ level $\left(n_{2}\right)$ and (d) the laser output, for a pump power $P_{p} \equiv 4 P_{p, h}$.

Figure 5 shows the behaviours obtained for the laser operation under different continuous pumping conditions. By using the above-mentioned parameters in the model resolution, we have observed that: at $P_{p}$-values larger than $P_{p, h}$ and

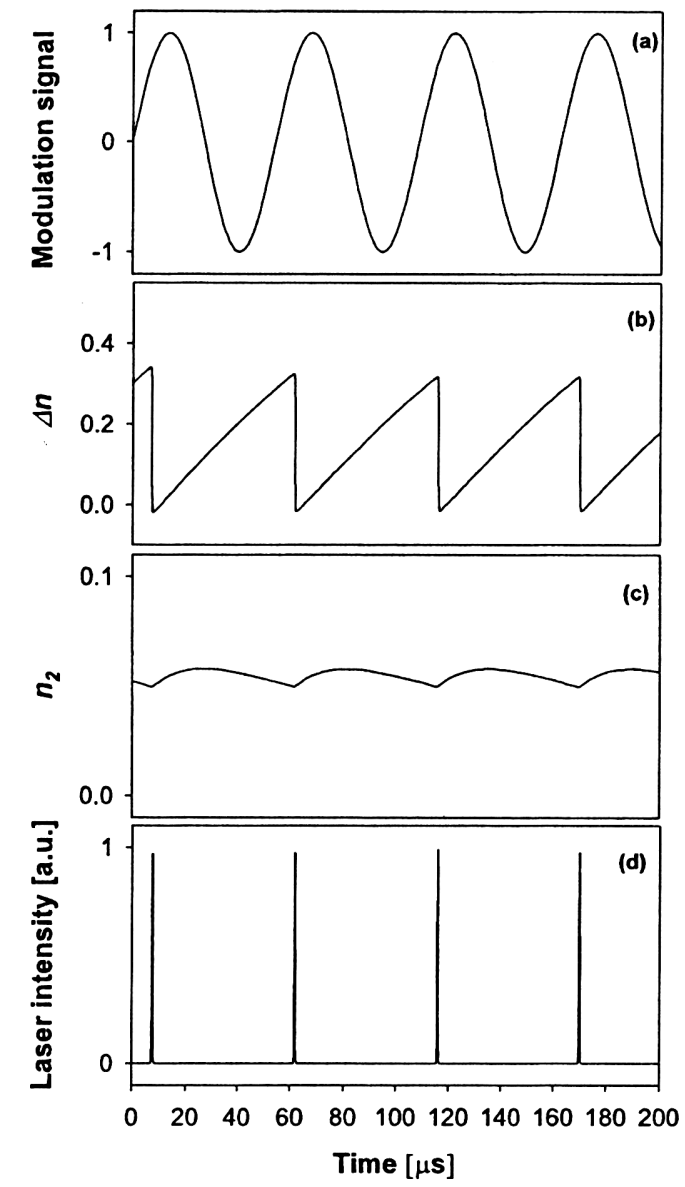

Figure 4. Numerical simulation with $P_{p} \equiv 4 P_{p, h}$ : (a) the normalized PZT modulation signal at $f_{0}=18.5 \mathrm{kHz}$; (b) population inversion for the laser transition $(\Delta n)$; (c) population of the ${ }^{4} I_{11 / 2}$ level $\left(n_{2}\right)$, and (d) normalized laser output.

smaller than $\sim 2 P_{p, h}$, lasing occurs in an unstable regime with a frequency $f<f_{0}$; at $\sim 2 P_{p, h}<P_{p}<\sim 6 P_{p, h}$, PZT governs the laser oscillation and we had a stable regime with $f \equiv f_{0}$; and when the pump excitation increases to above $6 P_{p, h}$, the laser output does not increase and the laser operation becomes unstable with the appearance of additional laser peaks $\left(f>f_{0}\right.$ ). The experimental limit for stable operation was found to be lower $\left(P_{p} \approx 5 P_{p . h}\right)$ than the theoretical one. The general characteristics described using this model are in agreement with the experimental behaviour of the Q-switched laser described in [7].

For comparison, figure 6 shows typical experimental pulsed laser emission, obtained with the laser system described in [7], as well as the modulating signal at $18.5 \mathrm{kHz}$ repetition rate measured for the PZT. Theoretical values of the temporal pulse width obtained from the model at $18.5 \mathrm{kHz}(\sim 1 \mu \mathrm{s})$ are close to the experimental ones. This value is approximately constant in the region of stable operation.

Figure 7 shows the spectral evolution of one of the pulses when the laser operates at $18.5 \mathrm{kHz}$. A very small frequency shift of the maximum occurs before a 'broadband' distribution is achieved. The temporal distributions (a), (b), (c), (d) and (e) correspond to the wavelengths 1540.20 , $1540.23,1540.26,1540.30$ and $1540.34 \mathrm{~nm}$, respectively. 


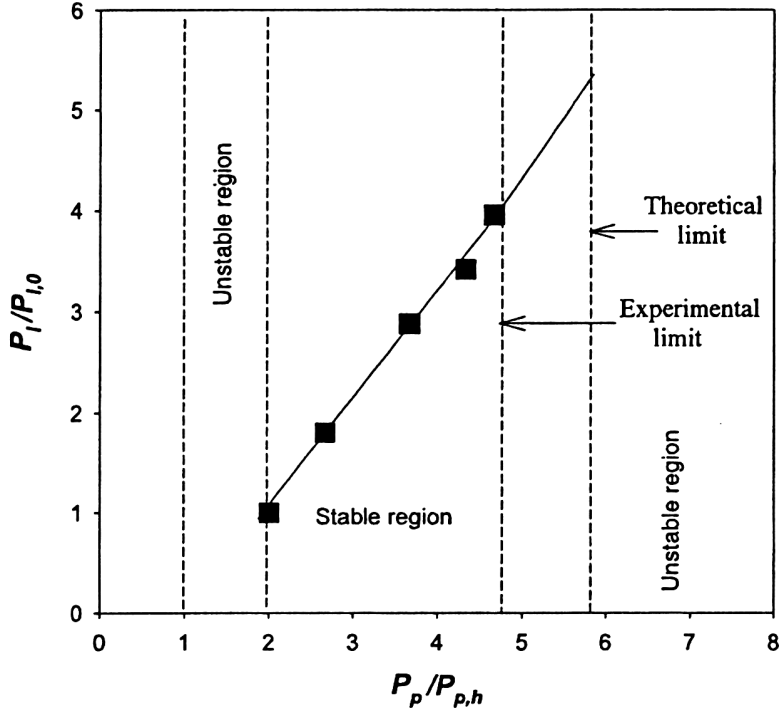

Figure 5. Laser output behaviour under different pump conditions. $P_{L}$ and $P_{L, 0}$ are the laser output power at an arbitrary pump power and with the lowest pumping for stable operation, respectively. The solid line represents the theoretical results and square points are experimental data.

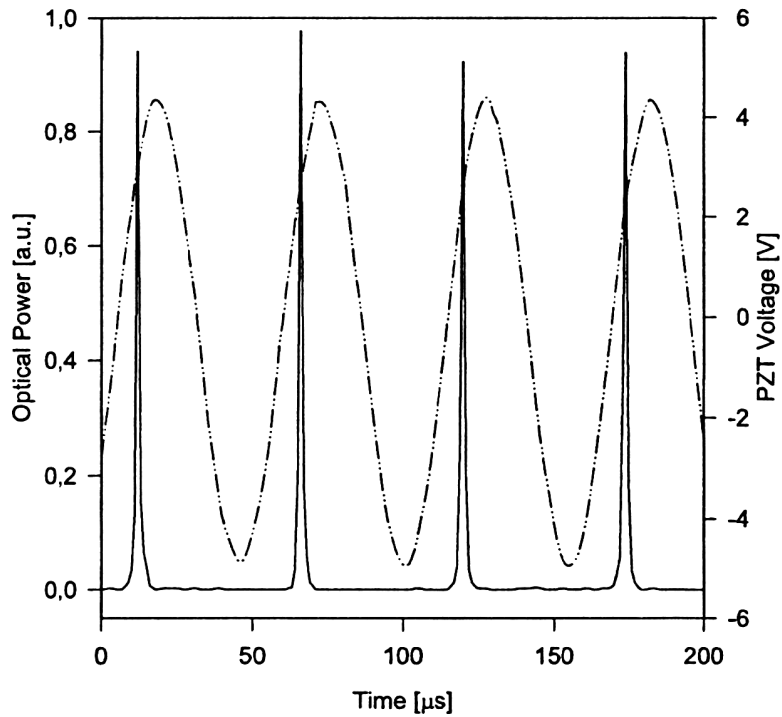

Figure 6. The experimental PZT modulation voltage and laser intensity evolution.

At larger frequencies $\left(f \gg f_{0}\right)$, the frequency shift starts to become more important and the pulses are chirped, due to the faster frequency oscillation of the cavity. A complementary experimental study of the spectro-temporal behaviour will be carried out in the near future. Figure 8 shows the agreement between the theoretical and the experimental spectral distributions obtained in the $1540 \mathrm{~nm}$ region for the emission of the $Q$-switched laser. The asymmetry observed in both curves appears also when the laser system operates at 1530 and $1554 \mathrm{~nm}$. The spectral pulse width depends on the spectral characteristics of both gratings and, particularly, on that of the grating with the narrow spectral width. Differences between theoretical and experimental curves are due to imperfections

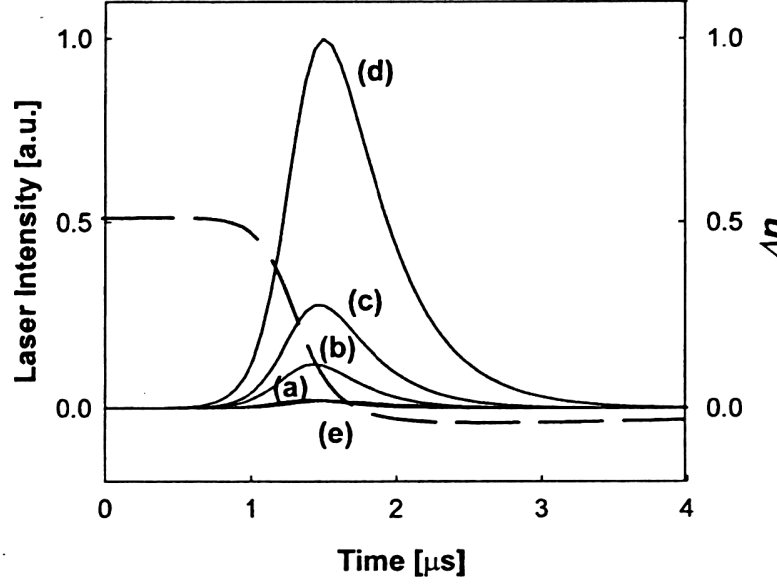

Figure 7. Evolution of the laser emission (theoretical) for several frequency components (solid curves): the temporal distributions of (a), (b), (c), (d) and (e) correspond to the wavelengths 1540.20 , $1540.23,1540.26,1540.30$ and $1540.34 \mathrm{~nm}$, respectively. The normalized population inversion is also shown (dashed curve).

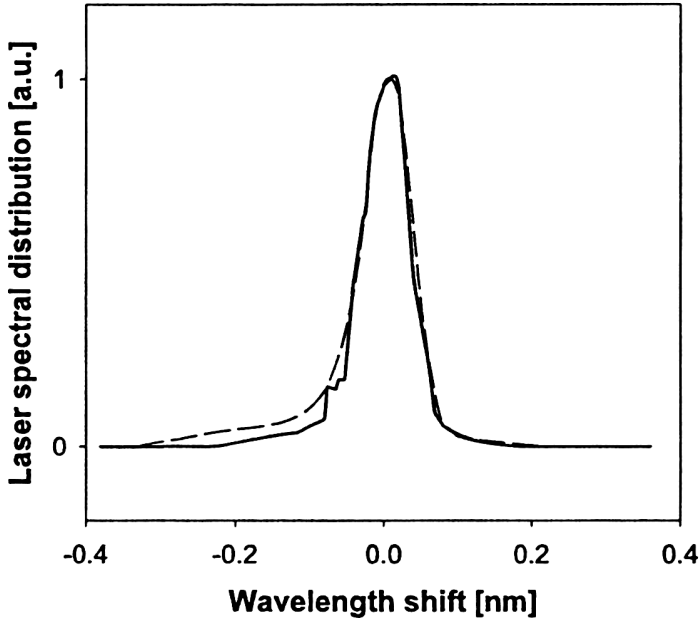

Figure 8. Spectral distributions of the laser emission: theoretical (dashed curve) and experimental (solid curve).

of the spectral grating distributions used in the experimental system.

In [7], the PZT was modulated by a square or sinusoidal voltage wave. In both cases, the laser system was always modulated at $f_{0} \equiv 18.5 \mathrm{kHz}$, because PZT works better at its resonance frequency. Also, other frequencies have harmonics coincident with this value $(18.5 \mathrm{kHz})$. Because of this, under continuous optical excitation and with square voltage pulses applied to the PZT, the laser behaves similarly for frequencies $f=f_{0} / n$ (where $n$ is an integer). When the pump excitation was also modulated, the excitation time could only be increased by $10-15 \%$ due to the PZT resonance. In these conditions, the laser output was increased by a factor $\sim 2$ and the temporal width was reduced by the same factor.

\section{Conclusions}

A Q-switched fibre laser system actively modulated by using a Bragg grating fixed to PZT was theoretically analysed employing a three-level scheme for the erbium-doped fibre and 
a wavelength dependence for the temporal modulation. In our model, we assume that the cavity length is large enough (some metres) that thousands of modes are simultaneously excited. Therefore the wave patterns between different waves can become sufficiently complex that many of the cross-coupling effects tend to be washed out on average, with the result that only the averaged intensity of all the waves is significant [11]. Results obtained with this scheme show several characteristics of the laser operation and they are in good agreement with experimental data previously reported [7].

\section{Acknowledgments}

This work was supported by the Consejo Nacional de Investigaciones Científicas y Técnicas (CONICET), the Universidad Nacional de La Plata (UNLP), the Agencia de Investigaciones Científicas, the Comisión de Investigaciones Científicas de la Provincia de Buenos Aires (CIC) and the Universidad Argentina de la Empresa (UADE, Buenos Aires) of Argentina; and by the Project AECI2001-IB-03 of Agencia Española de Cooperación Internacional (Spain).

\section{References}

[1] Desurvire E 1994 Erbium-Doped Fiber Amplifiers. Principles and Applications (New York: Wiley)

[2] Barnes W L 1993 Rare Earth Doped Fibre Lasers and Amplifiers ed M J F Digonnet (New York: Dekker) p 375

[3] Dominic V, MacCormack S, Waarts R, Stevens S, Bicknese S, Dohle R, Wolak E, Yeh P S and Zucker E 1999 CLEO'99: Conf. on Lasers and Electro Optics (Washington, DC: Optical Society of America) p 578

[4] Mizrahi V, DiGiovanni D J, Atkins R M, Grubb S G, Park Y K and Delavaux M P 1993 J. Lightwave Technol. 11 2021

[5] Sejka M, Varming P, Hubner J and Kristensen M 1995 Electron. Lett. 311445

[6] Russo N A, Duchowicz R, Mora J, Cruz J L and Andrés M V 2001 Proc. SPIE 441986

[7] Russo N A, Duchowicz R, Mora J, Cruz J L and Andrés M V 2002 Opt. Commun. 210361

[8] Imai T, Komukai T, Yamamoto T and Nakazawa M 1996 Japan. J. Appl. Phys. 235 L1275

[9] Lou L and Chu P L 1999 Opt. Commun. 161257

[10] Lou L G and Chu P L 1997 Opt. Lett. 221174

[11] Siegman A E 1986 Lasers (Oxford: Oxford University Press)

[12] Digonnet M J F 1990 IEEE J. Quantuin Electron. 261786

[13] Lou L G and Chu P L 1998 Opt. Commun. 149307 